\title{
Distribuição configurada pela artéria celíaca em papagaios-verdadeiros (Amazona aestiva)
}

\author{
[Configured distribution of the celiac artery in blue-fronted amazon (Amazona aestiva)] \\ E.S. Gonçalves ${ }^{1}$, M.I. Santana ${ }^{2 *}$, F.T. Zancan ${ }^{1}$, A.B. F. Pinto ${ }^{1}$, E.M.M. Lima ${ }^{2}$ \\ ${ }^{1}$ Medicina Veterinária - Pontifícia Universidade Católica de Minas Gerais - Poços de Caldas, MG \\ ${ }^{2}$ Laboratório de Anatomia Veterinária - Faculdade de Agronomia e Medicina Veterinária \\ Universidade de Brasília - ICC Ala Sul - Campus Universitário Darcy Ribeiro - Brasília - DF \\ ${ }^{3}$ Criadouro Científico e Cultural Poços de Caldas - Poços de Caldas, MG
}

\begin{abstract}
RESUMO
Foram descritas a origem e a distribuição da artéria celíaca em 20 papagaios-verdadeiros (Amazona aestiva), por meio do preenchimento do sistema arterial com solução de látex corado, fixado em solução de formol, para posterior dissecação de seus ramos colaterais. Como primeiro ramo, a artéria célica emitiu a artéria proventricular dorsal, que seguiu para o ventrículo como artéria gástrica dorsal e, em seguida, enviou a artéria proventricular ventral, que em $85 \%$ dos exemplares se originou diretamente da artéria celíaca, ou do seu ramo esquerdo em 15\%. Em 25\% dos casos, a artéria esplênica foi emitida antes da bifurcação em ramo esquerdo e direito. O ramo esquerdo foi responsável pela origem de ramos proventriculares, ramos pilóricos, artéria esplênica e artéria proventricular ventral, a qual emitiu ramos ao proventrículo e ao esôfago. A artéria celíaca também originou as artérias gástrica ventral e gástrica esquerda, que foram responsáveis pela emissão da artéria hepática esquerda. O ramo direito emitiu artérias esplênicas - que variaram de uma a sete -, duodenal destinada ao segmento mais caudal da alça ascendente do duodeno, hepática direita - para o lobo direito do fígado. Em seu segmento terminal, o ramo direito dividiu-se em artérias gástrica direita - para o ventrículo -, pancreaticoduodenal - para o pâncreas, alças ascendente e descendente do duodeno e segmentos cranial do íleo e caudal do jejuno. A irrigação determinada pela artéria celíaca nos papagaios segue o que é proposto para aves de outros gêneros, apresentando divergências como ausência das artérias vesicular e ileocecal, bem como da vesícula biliar e ceco.
\end{abstract}

Palavras-chave: papagaio-verdadeiro, anatomia, cavidade celomática, irrigação

\begin{abstract}
This research was performed aiming to describe the origin and the distribution of the celiac artery in 20 blue fronted amazon (Amazona aestiva), through the completion of their circulatory system with an aqueous solution of colored latex, followed by fixation in formol for later dissection of the collateral branches. The first branch emitted the proventricular dorsal artery that headed to the ventricle as gastric dorsal artery and next emitted the proventricular ventral artery which originated on the celiac artery in $85 \%$ of the cases and in 15\% had its origin on the left branch of the celiac artery. In 25\% of the cases the splenic artery was emitted before the bifurcation on left and right branches. The left branch was responsible for the origin of the proventricular branches, piloric branches, splenic artery and proventricular artery, which emitted branches to the proventricle and esophagus. The celiac artery also emitted the gastric ventral artery and left gastric. The right branch emitted splenic arteries (that varied from a number of seven); duodenal bound for the more caudal segment of the ascendant segment of the duodenum and the right hepatica to the right liver lobe. On its terminal segment the right branch was divided into right gastric arteries to the ventricle and pancreaticduodenal to the pancreas, ascendant and descendant braces of the duodenum and to the cranial segments of the ileum and caudal of the jejune. The irrigation provided by the celiac artery in parrots remains the same as described for other bird genders showing as divergences: the lack of vesicular arteries and ileocecal as well the gall bladder and the cecos.
\end{abstract}

Keywords: blue fronted amazon, anatomy, celomatic cavity, irrigation

Recebido em 18 de maio de 2010

Aceito em 30 de junho de 2011

E-mail: misantana@unb.br 


\section{INTRODUÇÃO}

Psitacídeos são aves encontradas em distintas regiões do globo terrestre, desde regiões de clima tropical até áreas com temperaturas amenas. Apresentam grande variação quanto ao tamanho, peso e coloração, e se caracterizam por apresentarem o bico curto de base larga e curvado, que as capacita a quebrar sementes duras e frutos (Sick, 1997; Godoy, 2007).

Na América do Sul e Central existem 27 espécies do gênero Amazona spp., sendo que o papagaioverdadeiro (Amazona aestiva) se destaca pela sua ampla distribuição natural e por possuir hábitos alimentares variados, além de ser muito procurado como ave de companhia, tanto por sua capacidade de imitar a voz humana como por sua beleza e docilidade (Wolvekamp, 2004).

Em levantamento realizado em Hospital Veterinário Escola no ambulatório de animais selvagens, entre os anos de 2003 e 2006, observou-se que a ordem dos psitaciformes foi a com maior número de casos atendidos, sendo o papagaio-verdadeiro a espécie responsável pelo segundo maior número de atendimentos (Santos et al., 2008a). No entanto, apesar da abundância de literatura anatômica encontrada para aves de espécies domésticas, esta ainda é escassa para a maioria das espécies pertencentes à avifauna brasileira, fato que tem gerado grande demanda para novos estudos descritivos (Perissoto e Silva, 2007). Sabe-se que o conhecimento morfológico é fundamental para fornecer subsídios à criação destas aves em cativeiro, a qual deve ser embasada em conhecimentos científicos (Santos et al., 2008b).

Dessa forma, o presente estudo teve os objetivos de estudar a origem e a distribuição da artéria celíaca e de seus ramos colaterais em papagaio verdadeiro e compará-la com a de outras espécies de aves domésticas e silvestres.

\section{MATERIAL E MÉTODOS}

Foram utilizados 20 papagaios-verdadeiros adultos, cinco fêmeas e 15 machos, pertencentes à espécie Amazona aestiva. As aves foram provenientes do Criatório Científico e Cultural de Poços de Caldas, MG (IBAMA - 2.31.94-00006), doadas após óbitos motivados por causa natural.

Para destaque do sistema circulatório, as aves tiveram a artéria isquiática direita canulada para injeção de solução de látex corado. Imediatamente após, foram fixadas em solução aquosa de formol a $10 \%$, acondicionadas em recipientes adequados e posteriormente dissecadas com material cirúrgico apropriado. Inicialmente, foram feitas duas incisões perpendiculares ao gradil costal dos dois antímeros, assim como na musculatura presente na extremidade distal do osso esterno. A musculatura peitoral das aves foi rebatida, expondo a cavidade celomática para realização da dissecação dos vasos sanguíneos do estudo. Concomitantemente à dissecação, foram elaborados desenhos esquemáticos (Fig. 3), onde foram registrados a origem, o número e a distribuição configurada pela artéria celíaca. A denominação das artérias seguiu como base os termos determinados pela Nômina Anatômica Aviária (Baumel, 1979).

\section{RESULTADOS}

A artéria celíaca originou-se da face ventral da aorta descendente toracoabdominal, representando seu primeiro ramo visceral, e estava direcionada para o antímero direito da cavidade celomática. A artéria celíaca emitiu a artéria proventricular dorsal como primeiro ramo colateral, que se continuou diretamente como artéria gástrica dorsal. Em sequência, a artéria proventricular ventral teve sua origem no tronco celíaco em 17 exemplares (85\%) e originou-se no ramo esquerdo da artéria celíaca em três aves (15\%).

Após a emissão dos ramos citados, a artéria celíaca bifurcou-se em ramos esquerdo e direito, em que: 1 - o ramo esquerdo, em todas as aves estudadas, emitiu a artéria gástrica ventral como primeiro colateral, para continuar diretamente como artéria gástrica esquerda, caracterizando-a como ramo terminal; 2 - em quatro papagaios (20\%), o ramo esquerdo também originou ramos pilóricos, e em sete exemplares (35\%) enviou ramos proventriculares, e, excepcionalmente, em um caso (5\%), um ramo proventricular foi originado pela artéria gástrica esquerda.

A artéria hepática esquerda, em 19 exemplares (95\%), teve sua origem a partir da artéria gástrica ventral. Um exemplar (5\%) também apresentou uma artéria hepática esquerda acessória, originada da artéria gástrica esquerda.

O ramo direito emitiu as artérias esplênicas como primeiros ramos colaterais em 13 exemplares (65\%), e, em sequência, enviou as artérias hepática direita e gástrica direita em todos os casos estudados (100\%), além dos ramos pilóricos em 
três casos (15\%), continuando-se diretamente como artéria pancreaticoduodenal.

Esta última originou a artéria ileal em 13 exemplares (65\%), a qual, em um caso (5\%), foi originada a partir do ramo direito da artéria celíaca. Em seu trajeto, a artéria pancreaticoduodenal também emitiu a artéria jejunal em 16 aves (80\%).

As artérias esplênicas também tiveram outros dois sítios de origem: o tronco celíaco em cinco exemplares (25\%) e o ramo esquerdo da artéria celíaca, em outras duas aves (10\%). Ainda, em cinco casos (25\%), os ramos pilóricos também tiveram sua origem na artéria gástrica direita.

Em seu trajeto, a artéria hepática direita também emitiu uma artéria duodenal em cinco exemplares (25\%), que também foi originada do ramo direito da artéria celíaca em três exemplares (15\%).

Quanto à descrição da distribuição e respectiva área de irrigação da artéria celíaca e seus ramos colaterais, as Fig. 1 e 2 são apresentadas para expor a relação entre as artérias e seus respectivos números de ramos enviados:
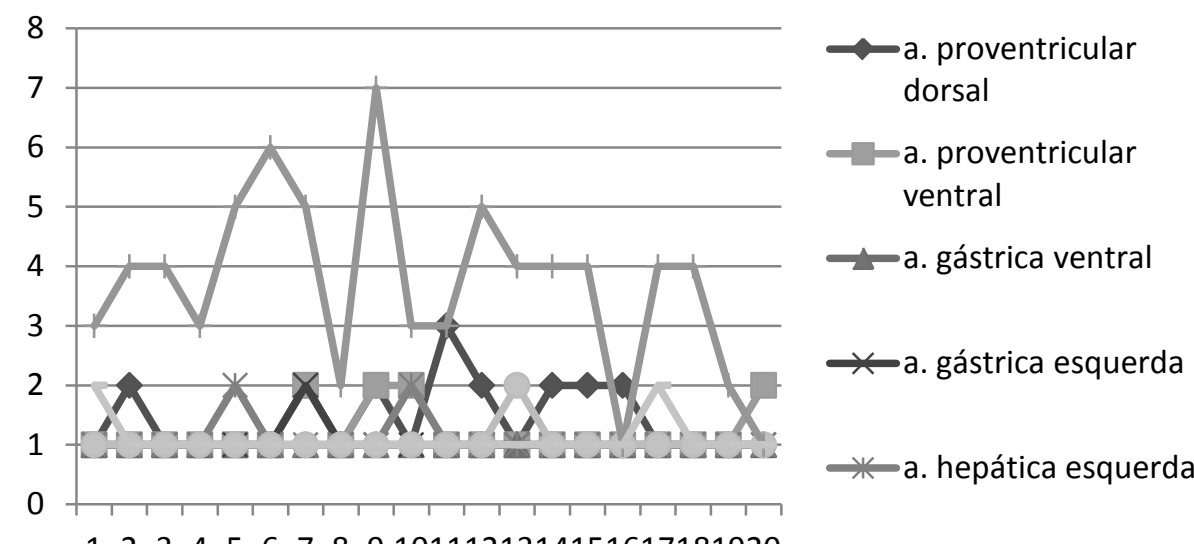

1234567891011121314151617181920

Figura 1. Distribuição dos ramos arteriais destinados a proventrículo, ventrículo, fígado e baço, em papagaios-verdadeiros (Amazona aestiva). A frequência foi determinada pelo número de ramos emitidos por artéria.

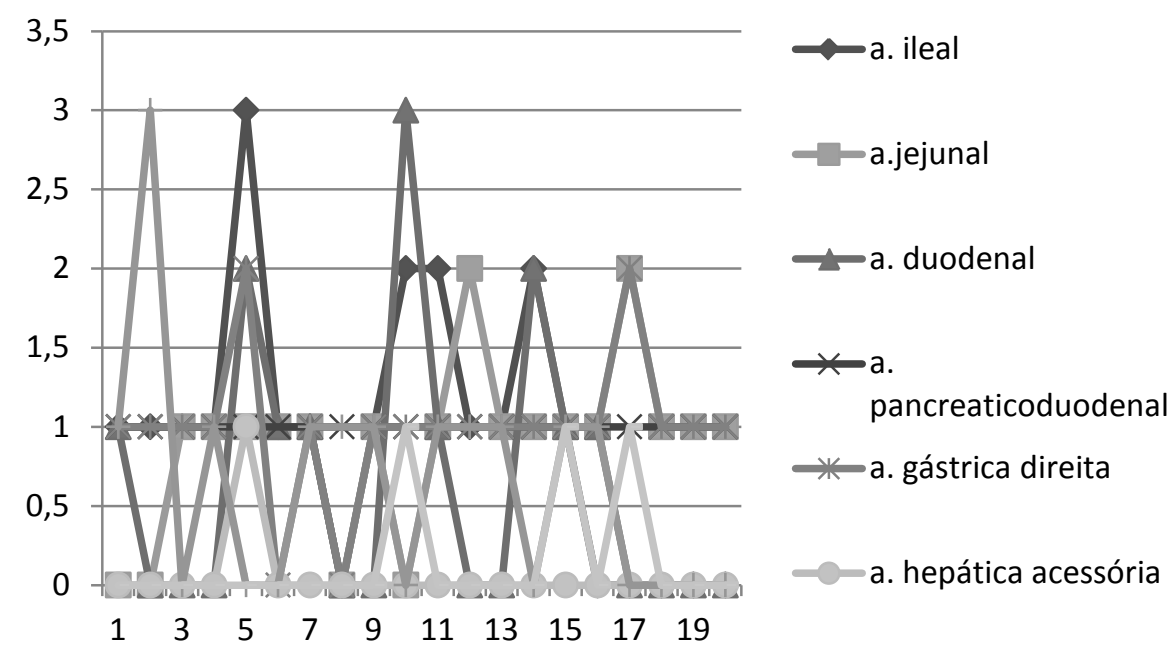

Figura 2. Distribuição dos ramos arteriais destinados a ventrículo, duodeno, jejuno, íleo, fígado e pâncreas, em papagaios-verdadeiros (Amazona aestiva). A frequência foi determinada pelo número de ramos emitidos por artéria. 


\section{Gonçalves et al.}

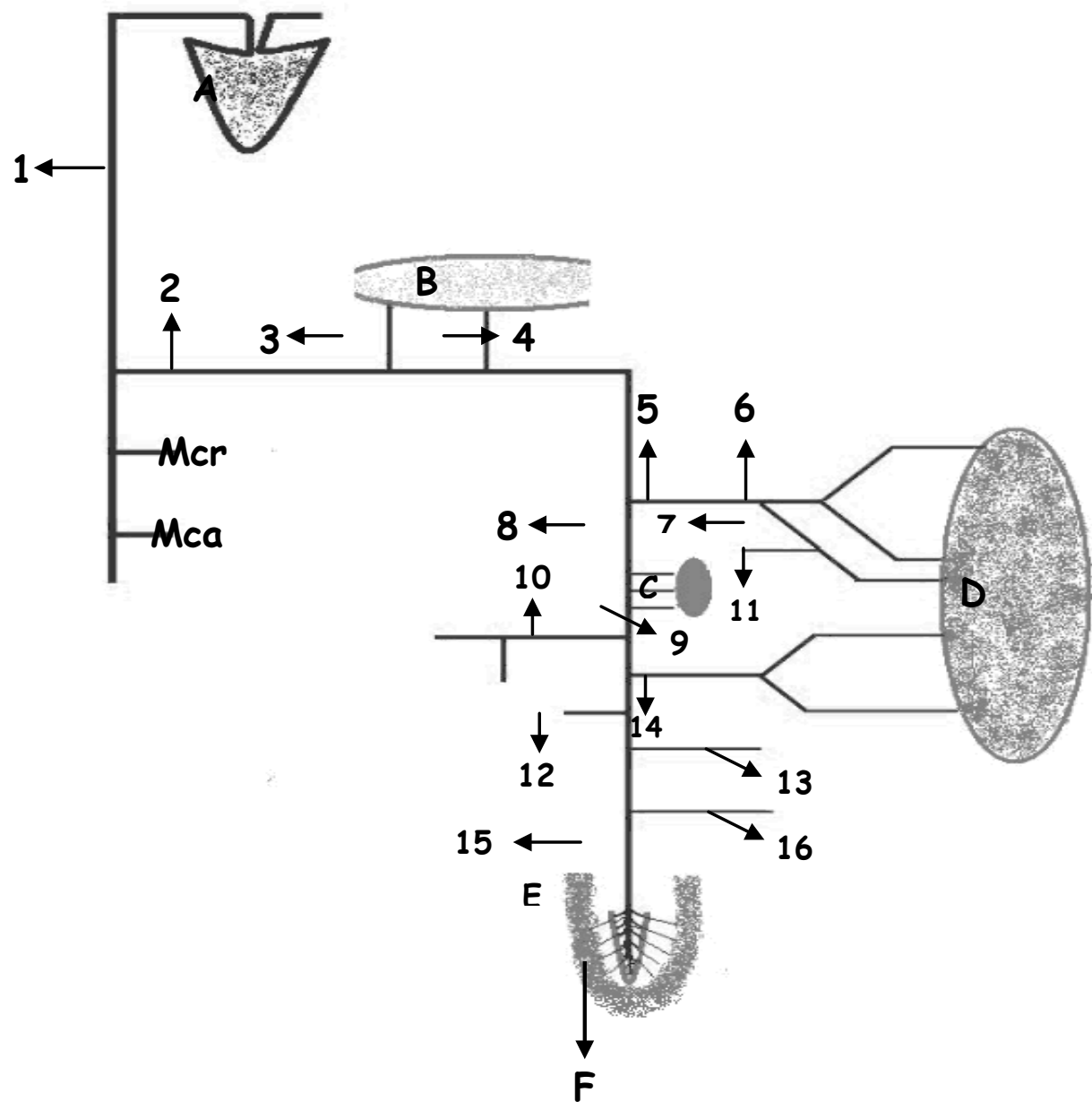

Figura 3. Esquema representativo da origem e distribuição dos ramos colaterais da artéria celíaca, em papagaiosverdadeiros (Amazona aestiva).

(A) coração; (B) proventrículo; (C) baço; (D) ventrículo; (E) duodeno; (F) pâncreas; (Mcr) A. mesentérica cranial; (Mca) A. mesentérica caudal; (1) Aorta descendente; (2) A. celíaca; (3) A. proventricular dorsal; (4) A. proventricular ventral; (5) ramo esquerdo da artéria celíaca; (6) A. gástrica esquerda; (7) A. gástrica ventral; (8) ramo direito da artéria celíaca; (9) A. esplênica; (10) A. hepática direita; (11) A. hepática esquerda; (12) A. duodenal; (13) A. ileal; (14) A. gástrica direita; (15) A. pancreaticoduodenal; (16) A. jejunal.

Ramo esquerdo da artéria celíaca: em quatro papagaios (20\%) irrigou diretamente a junção pilórica, através de ramos pilóricos, bem como enviou ramos proventriculares à porção ventral do proventrículo em sete casos (35\%).

Ramo direito da artéria celíaca: em três exemplares (15\%), enviou ramos pilóricos às porções cranial e caudal da junção pilórica.

Artéria proventricular dorsal: nos 20 exemplares, emitiu ramos para a porção dorsal do proventrículo.
Artéria proventricular ventral: emitiu ramos ao esôfago em dois exemplares (10\%) e em todos os exemplares, irrigou o proventrículo em sua porção ventral.

Artéria gástrica dorsal: presente em 100\% dos exemplares, sendo responsável pela irrigação da porção dorsal do ventrículo.

Artéria gástrica ventral: seu destino, em 100\% das aves estudadas, foi a porção ventral do ventrículo. 
Artéria gástrica esquerda: em 100\% dos papagaios estudados, foi responsável pela irrigação da face lateral esquerda do ventrículo, além de irrigar a porção ventral do proventrículo em um caso (5\%).

Artéria gástrica direita: em 100\% dos exemplares, foi responsável pela irrigação da face lateral direita do ventrículo, além de irrigar a porção ventral do proventrículo em cinco casos (25\%). Também emitiu ramos pilóricos à porção caudal da junção pilórica em cinco exemplares e, ainda, foi responsável pela irrigação da face direita do ventrículo, em 100\% dos papagaios.

Artéria hepática esquerda: em 17 casos (85\%), sua distribuição deu-se ao lobo hepático esquerdo.

Artéria hepática esquerda acessória: em um caso, colaborou com a irrigação do lobo hepático esquerdo.

Artérias esplênicas: o seu destino foi o baço em $100 \%$ dos papagaios.

Artéria hepática direita: em 100\% dos papagaios, sua distribuição foi o lobo hepático direito.

Artéria jejunal: seu destino foi o segmento mais caudal do jejuno em 16 aves (80\%).

Artéria duodenal: em oito casos (40\%), apresentou como área de irrigação exclusivamente o duodeno, especialmente o segmento mais caudal de sua alça ascendente.

Artéria ileal: sua área de irrigação foi o segmento cranial do íleo, em 14 papagaios (70\%).

Artéria pancreaticoduodenal: teve como destino, em $100 \%$ dos papagaios dissecados, o pâncreas e o segmento cranial do intestino delgado, especificadamente irrigando a alça ascendente $\mathrm{e}$ descendente do duodeno.

\section{DISCUSSÃO}

Existe uma carência de informações sobre a irrigação da cavidade celomática, determinada pela artéria celíaca, principalmente quando se trata da extensa avifauna brasileira, além do grande número de espécies exóticas introduzidas nos últimos anos. Sem dúvida, isto justifica a necessidade de desenvolver novos estudos morfológicos em aves silvestres, principalmente porque a grande maioria foi realizada em aves domésticas de interesse comercial (Perissoto e Silva, 2007).

Neste trabalho, notou-se que o padrão geral de vascularização determinado pelo tronco celíaco assemelha-se ao encontrado em estudos realizados com aves do gênero gallus (Malinovsky e Novotná, 1977; Getty, 1986; Silva et al., 1997; Silva et al., 2005), pato doméstico (Cairina moschata), murucututu (Pulsatrix perspicillata) e arara canindé (Ara ararauna) (Pinto et al., 1998; Silva et al., 2009 a, c). As semelhanças, no entanto, estão relacionadas diretamente à origem e às principais áreas de distribuição da artéria celíaca, pois, segundo Parizzi et al. (2000), Perissotto et al. (2001), Miranda et al. (2005) e Miranda e Silva (2007), essa artéria surge a partir da aorta descendente abdominal em sua porção ventral, enviando ramos para o baço, esôfago, proventrículo, ventrículo, fígado, duodeno, pâncreas e íleo.

Referente à distribuição da artéria celíaca, Malinovsky e Novotná (1977) informaram que esta fornece três ramos diretos, a artéria proventricular dorsal, o ramo esquerdo e o ramo direito, fato que, embora genérico, soma-se às observações deste trabalho e ao que foi encontrado em murucututus (Silva et al., 2009a), em suindaras (Tyto alba) (Silva et al., 2009d), no pato doméstico (Pinto et al., 1998) e em linhagens de galos e galinhas comerciais (Miranda et al., 2006).

No entanto, também foi encontrada variação quanto ao primeiro ramo enviado pela artéria celíaca, isto é, nos papagaios dissecados, no murucututu (Silva et al., 2009a), na skua (Stercorarius skua) (Silva et al., 2009b), na arara canindé (Silva et al., 2009c), na suindara (Silva et al., 2009d) e nas aves domésticas das linhagens Ross, Peterson, Redbro Plumé, Arbor Acres e Dekalb White (Silva et al., 1997; Cardoso et al., 2000; Miranda et al., 2005; Rafael et al., 2005; Miranda et al., 2006), a artéria proventricular dorsal foi computada como sendo o primeiro, enquanto em aves das linhagens Label Rouge, Cobb 500 e Label Rouge T44N (Perissoto et al., 2001; Silva et al., 2005; Perissoto e Silva, 2007), o primeiro foi a artéria esofágica. Este fato pode estar relacionado com 
variações referentes ao comprimento do esôfago entre as espécies e linhagens estudadas, além da possibilidade de a artéria esofágica não estar presente - como nos papagaios estudados - ou de ela se originar isoladamente da artéria celíaca (Perissoto et al., 2001; Silva et al., 2005; Perissoto e Silva, 2007) ou, ainda, como ramo direto da artéria proventricular dorsal (Silva et al., 2005), variando, dessa forma, sua posição como primeiro ramo da artéria celíaca.

Apenas uma artéria proventricular dorsal e ventral puderam ser identificadas na maioria das linhagens do gênero gallus (Miranda et al., 2005; Silva et al., 2005; Miranda et al., 2006; Miranda e Silva, 2007), no pato doméstico (Pinto et al., 1998), na suindara (Silva et al., 2009d) e em todos os papagaios dissecados, enquanto nas linhagens Peterson, Ross, Label Rouge, Arbor Acres e Label Rouge T44N foi encontrada mais de uma, apesar de os autores não informarem seu número exato (Cardoso et al., 2000; Silva et al., 2001; Perissoto et al., 2001; Rafael et al., 2005; Perissoto e Silva, 2007).

Adicionalmente a estes fatos, o proventrículo da espécie estudada foi um dos órgãos que apresentou maior variação de artérias a ele emitidas, pois, assim como nas linhagens Peterson, Label Rouge, Redbro Plumé, Cobb 500, Dekalb White e Label Rouge T44N e no pato doméstico (Pinto et al., 1998; Cardoso et al., 2000; Perissoto et al., 2001; Miranda et al., 2005; Silva et al., 2005; Miranda et al., 2006; Perissoto e Silva, 2007), recebeu ramos colaterais oriundos das artérias proventriculares dorsal e ventral e dos ramos esquerdo e direito da artéria celíaca.

No papagaio-verdadeiro também foi observada irrigação da porção ventral do proventrículo por ramos proventriculares oriundos das artérias gástricas esquerda e direita, fato não relatado na literatura consultada. Essas variações podem estar ligadas à eficiência alimentar, pois a ave que apresenta apenas um ramo chegando ao proventrículo, provavelmente, terá menor capacidade de síntese enzimática, quando comparada à que apresenta 10 ramos. Tais variações podem estar associadas à sintopia entre as artérias e o proventrículo, pois quanto mais próxima estiver a artéria de um órgão, mais fácil será a emissão de ramos diretos.
Segundo Cardoso et al. (2000), Perissoto et al. (2001), Miranda et al. (2005), Rafael et al. (2005), Silva et al. (2005), Miranda et al. (2006), Miranda e Silva (2007), assim como nos papagaios estudados, o ramo esquerdo originou as artérias gástricas ventral e esquerda e ramos proventriculares e pilóricos.

Foi observada diferença para a origem da artéria hepática esquerda, pois, nos papagaios dissecados e em araras canindé (Silva et al., 2009c), a origem se deu pela artéria gástrica ventral, ao invés de ser diretamente do ramo esquerdo, como nas diferentes linhagens de galos e galinhas descritas, o que pode representar uma característica inerente aos psitacídeos.

O ramo esquerdo originou, nas linhagens Cobb 500 e Bovans Goldline (Silva et al., 2005; Miranda e Silva, 2007), uma artéria esplênica, e nas linhagens Ross e Arbor Acres (Silva et al., 1997; Rafael et al., 2005), um ramo pericárdico, bem como emitiu a artéria proventricular ventral na linhagem Label Rouge (Perissoto et al., 2001) e duodenal na linhagem Cobb 500 (Silva et al., 2005), fatos estes que não tiveram comparação com o trabalho realizado e que, pela baixa ocorrência descrita, podem ser caracterizados como variações individuais das linhagens citadas.

Nos papagaios dissecados, o ramo direito, à semelhança dos descritos por Pinto et al. (1998) e Rafael et al. (2005), emitiu as artérias esplênicas, hepática direita, gástrica direita e ramos pilóricos e, diferentemente de Perissoto et al. (2001), Miranda et al. (2005), Miranda et al. (2006) e Perissoto e Silva (2007), não foi observada a emissão da artéria duodenal, que se originou da artéria hepática direita.

Também chamou a atenção o fato de todos os papagaios avaliados não terem apresentado vesícula biliar e cecos e, portanto, não foram evidenciados ramos císticos e artéria ileocecal, como descrito por Cardoso et al. (2000) e Silva et al. (2005), sendo encontrada, portanto, apenas a artéria ileal.

Quanto ao baço, Pinto et al. (1998) e Perissoto et al. (2001) ressaltaram que foi irrigado exclusivamente por artérias esplênicas originadas do ramo direito. Porém, nos animais deste trabalho, o baço também foi irrigado por artérias 
esplênicas originadas tanto do ramo direito quanto do ramo esquerdo e por aqueles originados do tronco celíaco. Esta variação pode estar relacionada com o tamanho avantajado deste órgão nos papagaios e pela pequena distância encontrada entre ele e as artérias supracitadas.

Outro ponto de divergência referiu-se à irrigação do segmento inicial do duodeno, que foi realizada pela artéria duodenal nos papagaios trabalhados, pela artéria duodenojejunal em araras canindé (Silva et al., 2009c) e nas linhagens Peterson, Redbro Plumé, Arbor Acres, Dekalb White e Bovans Goldline (Cardoso et al., 2000; Miranda et al., 2005; Rafael et al., 2005; Miranda et al., 2006; Miranda e Silva, 2007), e pela artéria pancreaticoduodenal no murucututu (Silva et al. 2009a), na skua (Silva et al., 2009b), nas linhagens Arbor Acres (Rafael et al., 2005) e Cobb 500 (Silva et al., 2005) e no pato doméstico (Pinto et al., 1998).

Talvez, esta variação possa ser entendida pela grande proximidade que este segmento intestinal tem com o ramo direito da artéria celíaca, o principal emissor das artérias duodenal, duodenojejunal e pancreaticoduodenal, podendo caracterizar este evento como aleatório e de caráter individual. Corroborando esta ideia, pode-se incluir a presença individual da artéria jejunal, encontrada tanto nos papagaios dissecados quanto nas linhagens Ross, Label Rouge, Cobb 500 e Label Rouge TN44 (Perissoto et al., 2001; Silva et al., 2005; Perissoto e Silva, 2007). Ainda, diferentemente do que foi relatado por Perissoto et al. (2001), Rafael et al. (2005), Silva et al. (2005), Miranda e Silva (2007) e Perissoto e Silva (2007), não foram encontradas anastomoses entre ramos das artérias jejunal e mesentérica cranial.

\section{CONCLUSÕES}

A origem e a forma de distribuição da artéria celíaca em Amazona aestiva segue o que é proposto para aves do gênero Gallus gallus domesticus, Cairina moschata, Stercorarius skua, Pulsatrix perspicillata, Tyto alba e Ara ararauna. A espécie Amazona aestiva apresenta algumas características próprias, como ausência em $100 \%$ dos casos estudados das artérias esofágica, ileocecal e ramos císticos, bem como da vesícula biliar e dos cecos. A ausência destas estruturas provavelmente ocorreu ao longo da evolução da espécie e pode estar associada à dieta carente em fibras e à alta taxa de passagem. Variações com relação à distribuição e ao número de ramos colaterais emitidos pela artéria celíaca também foram observadas e podem estar associadas à sintopia entre artéria e órgão e/ou à eficiência alimentar de cada ave.

\section{REFERÊNCIAS BIBLIOGRÁFICAS}

BAUMEL, J.J. Nomina anatomica avium. London: Academic, 1979. p.360-361.

CARDOSO, J.R.; DRUMMOND, S.S.; MARTINS, A.K. et al. Aspectos anatômicos da artéria celíaca em Gallus gallus da linhagem Peterson. Arq. Cien. Vet. Zool. UNIPAR, v.3, p.151-157, 2000.

GETTY, R. Sisson-grossman anatomia dos animais domésticos. 5.ed. Rio de Janeiro: Guanabara Koogan, 1986. v.2, p. 1862-1863.

GODOY, S.N. Psitaciformes (Arara, Papagaio, Periquito). In: CUBAS, Z.S.; SILVA, J.C.R.; DIAS, J.L.C. Tratado de animais selvagens medicina veterinária. São Paulo: Roca, 2007. Cap. 16, p.222223.

MALINOVSKY, L.; NOVOTNÁ, M. Branching of the coeliac artery in some domestic birds III. A comparison of the pattern of the coeliac artery in three breeds of the domestic fowl (Gallus gallus domesticus). Anat. Anz., v.141, p.136-146, 1977.

MIRANDA, R.L.; SILVA, F.O.C. Origem e distribuição da artéria celíaca em aves (Gallus gallus) poedeiras de linhagem Bovans Goldline. Rev. Horiz. Cient., v.1, p. 1-15, 2007.

MIRANDA, R.L.; SILVA, F.O.C.; LIMA, J.C.S. et al. Origem e distribuição da artéria celíaca em aves (Gallus gallus) de corte da linhagem Redbro Plumé. Biosci. J., v.21, p.77-83, 2005.

MIRANDA, R.L.; SILVA, F.O.C.; SEVERINO, R.S. et al. Origem e distribuição da artéria celíaca em aves (Gallus gallus) poedeiras da linhagem Dekalb White. Vet. Not., v.12, p.41-46, 2006.

PARIZZI, A.; GONÇALVES, P.R.; MACHADO, G.V. et al. Origem e distribuição da artéria celíaca em flamingos (Phoenicopterus ruber chilensis). Braz. J. Vet. Res. Anim. Sci., v.17, p.86-87, 2000.

PERISSOTTO, D.O.; SILVA, F.O.C. Origem e distribuição da artéria celíaca em aves (Gallus gallus) da linhagem Label Rouge T44 N. Rev. Horiz. Cient., v.1, p.1-15, 2007. 


\section{Gonçalves et al.}

PERISSOTTO, D.O.; SILVA, F.O.C.; SEVERINO, R.S. et al. Origem e distribuição da artéria celíaca em aves Gallus gallus (matrizes de corte -linhagem Label Rouge). Arq. Cien. Vet. Zool. UNIPAR, v.4, p.155161, 2001.

PINTO, M.R.A.; RIBEIRO, A.A.C.M.; SOUZA, W.M. Os arranjos configurados pela artéria celíaca no pato doméstico (Cairina moschata). Braz. J. Vet. Res. Anim. Sci., v.35, p.103-106, 1998.

RAFAEL, E.L.S.; SILVA, F.O.C.; SEVERINO, R.S. et al. Origem e distribuição da artéria celíaca em aves (Gallus gallus Linnaeus 1758) da linhagem Arbor Acres. Biosci. J., v.21, p.55- 60, 2005.

SANTOS, G.C.; MATUELLA, G.A.; CARAIOLA, A.M. et al. Doenças de aves selvagens diagnosticadas na Universidade Federal do Paraná (2003-2007). Pesq. Vet. Bras., v.38, p.565-570, 2008a.

SANTOS, T.; TRUJILlO, H.A.G.; ALBERTO, M.L.V. et al. Aspectos morfológicos e morfométricos da traqueia em gansos domésticos. Biotemas, v.21, p.91-96, 2008b.

SICK, H. Ornitologia brasileira. 2.ed. Rio de Janeiro: Nova Fronteira, 1997. p.351-379.

SILVA, A.C.J.; BARROS, M.L.G.; NASCIMENTO, H.B. et al. Origem e distribuição da artéria celíaca em murucututu (Pulsatrix perspicillata). In: ENCONTRO INTERNACIONAL DE MEDICINA DA CONSERVAÇÃO. MUDANÇAS CLIMÁTICAS, SAÚDE E ECOSSISTEMAS: A SAÚDE NUMA DIMENSÃO ECOLÓGICA, 2., 2009, Recife, PE. Anais..., Recife: UFRFE, 2009a. p.119.

SILVA, A.C.J.; SILVEIRA, B.M.R.; JUNIOR, F.P.N. et al. Descrição das artérias celíaca, mesentérica cranial e mesentérica caudal em skua (Stercorarius skua). In: ENCONTRO INTERNACIONAL DE MEDICINA DA CONSERVAÇÃO. MUDANÇAS CLIMÁTICAS, SAÚDE E ECOSSISTEMAS: A SAÚDE NUMA DIMENSÃO ECOLÓGICA, 2., 2009, Recife, PE. Anais..., Recife: UFRFE, 2009b. p.108.
SILVA, A.C.J.; QUEIROZ, R.A.; BANDEIRA, J.T. et al. Estudo descritivo das artérias celíaca, mesentérica cranial e mesentérica caudal em arara Canindé (Ara ararauna) In: ENCONTRO INTERNACIONAL DE MEDICINA DA CONSERVAÇÃO. MUDANÇAS CLIMÁTICAS, SAÚDE E ECOSSISTEMAS: A SAÚDE NUMA DIMENSÃO ECOLÓGICA, 2., 2009, Recife, PE. Anais..., Recife: UFRFE, 2009c. p.110.

SILVA, A.C.J.; BARROS, M.L.G.; NASCIMENTO, H.B. et al. Origem e distribuição da artéria celíaca em suindara (Tyto alba). In: ENCONTRO INTERNACIONAL DE MEDICINA DA CONSERVAÇÃO. MUDANÇAS CLIMÁTICAS, SAÚDE E ECOSSISTEMAS: A SAÚDE NUMA DIMENSÃO ECOLÓGICA, 2., 2009, Recife, PE. Anais..., Recife: UFRFE, 2009d. p.118.

SILVA, F.O.C.; SEVERINO, R.S.; SANTOS, A.L.Q. et al. Origem, ramificações e distribuições da artéria celíaca em aves fêmeas (Gallus gallus) da linhagem Cobb 500. Biosci. J., v.21, p.149-154, 2005.

SILVA, F.O.C.; SEVERINO, R.S.; SANTOS, A.L.Q. et al. Origem e distribuição da artéria celíaca em aves (Gallus gallus domesticus linhagem Ross). Rev. FZVA, v.4, p.35-41, 1997.

WOLVEKAMP, P. Radiography of Birds and Exotic Animals. In: WORLD SMALL ANIMAL VETERINARY ASSOCIATION CONGRESS, 29., 2004, Rhodes, Grece. Proceedings ..., Rhodes: WSAVA, 2004. p.1-3. 